\title{
Revue des livres
}

\section{Radiation Protection and Safety of Radiation Sources: International Basic safety}

Standards, Interim edition, ISBN 978-92-0-120910-8, IAEA, Vienne 2011, $65 €$.

Elles étaient attendues, voici les nouvelles « Basic Safety Standards » publiés par l'AIEA. Les BSS précédents publiés en 1996, ont fait l'objet d'une révision importante, commencée en 2007, peu avant la parution de la publication 103 de la CIPR en 2007. Ces BSS intègrent également la décision prise par la CIPR en 2011 de réviser les doses limites pour le cristallin. Elle portent le nom de d'édition intérimaire dans l'attente de leur validation par les organismes partenaires, OCDE/AEN, WHO, etc. Le texte imprimé en anglais est accompagné d'un CD incluant les traductions en langues arabe, chinoise, française, russe et espagnol.

Les leçons tirées de l'accident de Fukushima feront éventuellement l'objet d'une version révisée dans le futur.

\section{H. Métivier} SFRP

\section{Recommandations de bonne pratique : surveillance médico-professionnelle de l'exposition interne aux radionucléides en installations nucléaires de base, 978-2- 7598-0729-1.}

Ce guide est un ouvrage collectif finalisé en 2011, de toute première importance, et c'est pourquoi la rédaction n'a pas hésité à demander à certains de ses auteurs et à un expert critique de nous donner leur point de vue. Il fait l'unanimité. Ce guide peut être téléchargé à partir du site de la Société Française de Médecine du Travail : http://www.chu-rouen.fr/sfmt/pages/ accueil.php

\section{Le point de vue de Philippe Bérard, Nicolas Blanchin, Michèle Gonin, Benoît Quesne}

En France, 64000 travailleurs d'installations nucléaires de base (INB) sont susceptibles d'être exposés au risque de contamination interne par des radionucléides. Leur suivi est assuré par 450 médecins du travail habilités, exerçant dans des services autonomes ou des services interentreprises. Un groupe de travail a été créé, en 2004, à l'initiative de médecins du travail de différentes INB pour échanger leurs interrogations à propos de la dosimétrie interne réalisée chez ces travailleurs, et notamment lister les difficultés dans le but d'harmoniser leurs pratiques. De cette initiative est né un guide de bonnes pratiques, pouvant servir de référentiel aux médecins du travail concernés par ce risque, mais également aux médecins hospitaliers et urgentistes susceptibles de prendre en charge des victimes d'une contamination radioactive. Ce guide a été réalisé sous la promotion de la Société Française de Médecine du Travail (SFMT). Il a été rédigé par un groupe de professionnels, composé de médecins du travail, de 
REVUE DES LIVRES

biologistes médicaux et d'experts en dosimétrie interne. La rédaction de ce guide suit la méthodologie définie par la Haute Autorité de Santé (HAS). Sa finalité est d'émettre des recommandations basées sur les connaissances scientifiques et le retour d'expérience des pratiques professionnelles dans le domaine de la dosimétrie interne. Le respect scrupuleux des méthodes conseillées par la HAS, pour l'élaboration de recommandations médicales, a permis au guide d'obtenir le label de cet organisme officiel.

\section{Le point de vue de Laurence Lebaron-Jacobs}

Optimiser la prévention du risque d'exposition interne et le suivi médical des travailleurs exposés à ce risque a toujours été au cœur des interrogations entre médecins du travail des installations nucléaires de base (INB). En 2004, ils décident de créer un groupe de travail et se fixent trois objectifs :

- homogénéiser les pratiques professionnelles de médecin du travail ;

- renforcer la prévention primaire en contribuant à l'amélioration de la propreté radiologique des postes de travail en coordination avec les autres acteurs de la prévention en santé au travail et la prise en compte du retour d'expérience ;

- améliorer les actions d'information auprès des travailleurs sur la nature des risques auxquels ils sont exposés.

En effet, la réglementation française attribue la responsabilité de la surveillance individuelle de l'exposition interne et de l'estimation dosimétrique et sanitaire aux médecins du travail des INB. Cependant, comme la littérature ne foisonne pas de publications sur les modalités opérationnelles de la surveillance individuelle en INB, la mise en place de programmes de surveillance ainsi que la compréhension et l'appropriation des méthodes d'évaluation restent un exercice difficile.

De cette réflexion est né un guide de bonnes pratiques promu par la Société Française de Médecine du Travail (SFMT), pouvant servir de référentiel aux médecins du travail des INB, mais également aux médecins hospitaliers et urgentistes susceptibles de prendre en charge des victimes d'une contamination interne. Ce guide a été rédigé par un groupe de professionnels, composé de médecins du travail, de biologistes médicaux et d'experts en dosimétrie interne en tenant compte des exigences de la méthode d'élaboration des recommandations pour la pratique clinique de la Haute Autorité de Santé (HAS). L'intérêt de cette méthode est d'associer les connaissances scientifiques au retour d'expérience des pratiques professionnelles en dosimétrie interne.

La spécificité de la réglementation française dans ce domaine limite ce guide de bonnes pratiques uniquement au champ médico-professionnel du secteur nucléaire en INB. Cependant, il pourra servir de base à l'élaboration de recommandations pour le secteur médical, la recherche et les industries non nucléaires. Par ailleurs, les recommandations ciblent les expositions par inhalation, qui correspondent aux modalités d'exposition majoritaire des travailleurs. À noter que la problématique d'une exposition par plaie contaminée, pouvant induire une dose locale puis une dose liée au transfert direct au niveau systémique, est tout de même abordée. En revanche, les expositions liées à l'ingestion de produits contaminés, correspondant aux modalités d'exposition principale des populations, ne sont pas traitées. 
Enfin, ces recommandations sont limitées aux principaux radionucléides à l'origine des expositions professionnelles en INB.

Les médecins du travail des INB espéraient tous qu'un jour ils pourraient aborder de façon plus sereine la prévention du risque d'exposition interne et le suivi médical des travailleurs exposés. C'est chose faite grâce aux recommandations de bonne pratique de ce guide « Surveillance médico-professionnelle de l'exposition interne aux radionucléides en installations nucléaires de base », basées sur la réglementation et les prescriptions normatives, sur les connaissances scientifiques et sur le retour d'expérience des pratiques professionnelles.

Mesure du rayonnement alpha, CETAMA - Dossier de recommandations pour l'optimisation des mesures, Eric Ansoborlo, Jean Aupiais, Nicolas Baglan, coordonnateurs, Lavoisier, 2012, ISBN 978-2-7430-1414-8.

Utilisé dans le domaine médical, présent dans l'industrie militaire ou encore électronucléaire, le rayonnement alpha constitue un des principaux rayonnements dont la détection et la mesure sont primordiales, que ce soit pour le contrôle de l'environnement, le suivi de toute opération technique industrielle ou la mesure de l'impact de la radioactivité sur la santé. Cet ouvrage rassemble les connaissances de base, aussi bien pratiques que théoriques, ainsi que toutes les recommandations nécessaires à tout opérateur, technicien ou ingénieur souhaitant utiliser la spectrométrie alpha comme méthode d'analyse quantitative et qualitative tout en lui évitant tout écueil méthodologique et biais expérimental.

Il apporte ainsi des informations dans le domaine :

- de la préparation des sources pour la mesure par spectrométrie alpha ;

- de la mise en œuvre des mesures pour chaque technique : compteur proportionnel, spectrométrie alpha avec semi-conducteur, chambre à grille et scintillation liquide ;

- du traitement et de l'expression des résultats ;

- de l'assurance de la qualité.

Ainsi, ce livre synthétise le savoir-faire des laboratoires dans la fabrication des sources minces en donnant les règles simples mais nécessaires ainsi que des informations permettant d'appréhender les limites physiques et chimiques des sources minces. Il liste les techniques relatives à la détection des émissions alpha en décrivant, pour chacune d'elles, la sensibilité, les avantages et inconvénients, le champ d'action. Ce livre apporte aussi de manière aussi simple que possible les outils mathématiques requis pour déconvoluer les spectres alpha selon les techniques utilisées et donne des exemples de traitement qui permettent d'appréhender la complexité des cas rencontrés. Il fournit à l'utilisateur les bases pratiques et théoriques indispensables à la production de résultats fiables et validés. Il inclut et discute les dernières réflexions dans le calcul des seuils de décision et limites de détection.

P. Bérard

CEA 
Les réacteurs nucléaires expérimentaux, Une monographie de la direction de l'énergie nucléaire du CEA, Éditions le moniteur, 2012, ISBN 978-2-281-11509-3, $18 €$.

Voici le septième numéro de ces monographies que dirige notre ami Bernard Bonin au sein de la direction de l'énergie nucléaire du CEA. Aucune surprise avec ce volume, la qualité est toujours présente, la réalisation parfaite, la lecture de ces monographies est toujours un plaisir. Radioprotectionniste je me suis un peu plus attardé sur le réacteur Silène car il a permis aux équipes du département de Protection sanitaire de l'ex CEA/IPSN et du Centre de recherches de santé des armées d'étudier les effets biologiques, aussi bien déterministes que stochastiques de neutrons. Ce réacteur qui pouvait apporter aux chercheurs un flux de neutrons plus ou moins associé à un flux de rayonnement gamma, le tout parfaitement calibré, a permis aux équipes françaises de rivaliser avec les chercheurs américains sur cet important sujet. Mais en plus des études biologiques, ce réacteur est un outil unique au monde pour des intercomparaisons dosimétriques rassemblant des dizaines d'équipes internationales.

Nous attendons avec impatience le prochain numéro de ces monographies.

\section{H. Métivier} SFRP

Dosimétrie externe, Applications à la radioprotection, Alain Faussot, Lavoisier, 2011, ISBN 978-2-7430-1395-0.

Cet ouvrage de référence rassemble toutes les informations nécessaires pour comprendre et maîtriser la dosimétrie externe et la métrologie des rayonnements ionisants, depuis les effets des rayonnements jusqu'à l'étalonnage du matériel de radioprotection. Des exercices d'application accompagnés de leur corrigé sont proposés à la fin de chaque chapitre ; une excellent initiative.

Cet ouvrage est destiné aux étudiants en BTS de radioprotection, licence technique, masters 1 et 2, écoles d'ingénieurs mais aussi aux professionnels désireux d'actualiser leurs connaissances. Avec moins de 200 pages, cet ouvrage se glissera parfaitement dans votre bibliothèque, je vous le recommande.

H. Métivier SFRP

\section{Parmi les livres d'une brulante activité, citons :}

Le crédo antinucléaire, pour ou contre ? Pierre Bacher, Préface d'Édouard Brézin, Odile Jacob, 2012, ISBN 9782738 128195, 15,90€.

L'accident de Fukushima a ravivé la discussion autour de la poursuite ou non du nucléaire, mais les arguments n'ont pas changé. Pire, on ravive en les multipliant les conséquences de l'accident de Tchernobyl, le dernier en date pour une association antinucléaire était encore il $\mathrm{y}$ a quelques semaines, un bilan de 1 million de morts suite à cet accident. Ces associations font des déclarations de foi sur tous les sujets qui entourent le nucléaire, sans écouter le moins du monde les arguments des ingénieurs et des experts. Pierre Bacher, dans son court ouvrage 
(165 pages), démonte soigneusement les articles de foi du crédo antinucléaire et nous donne les cartes pour évaluer le pour et le contre, afin que chacun puisse se forger une opinion.

Un livre clair à lire et à faire lire au moment où le débat sur le nucléaire repart. Je rêve que chacun s'informe complètement avant d'asséner des propos définitifs sur une énergie, qu'on le veuille ou non, qui fait partie du mix énergétique qui nous sauvera du réchauffement climatique.

L'accident majeur de Fukushima, Considérations sismiques, nucléaires et médicales, Groupe de travail Solidarité Japon présidé par Alain Carpentier, président de l'Académie des sciences, EDP Sciences, 2012, ISBN 978-2-7598-0736-9, 112 pages avec $\mathrm{CD}, 29 €$

Contactée par son équivalente japonaise, l'Académie des sciences a organisé trois groupes de travail sur les « Mégaséismes et tsunami », «L'accident nucléaire » lui-même et sur «Les conséquences sanitaires et environnementales » de l'accident. Rassemblant les meilleurs experts dans leurs domaines, ce rapport concis (moins de 100 pages et un $\mathrm{CD}$ où l'ont peut retrouver tous les éléments qui ont permis d'écrire cette synthèse) est accompagné de recommandations claires. Quelles leçons en tirer? Bien des incertitudes demeurent notamment la réhabilitation des sols et la réintégration des populations déplacées, l'organisation optimale des soins et des mouvements de populations lors d'accident d'étendue et de gravité extrêmes qu'ils soient nucléaires ou pas.

Ce document s'adresse à la communauté scientifique internationale et tout spécialement à la communauté japonaise en témoignage de solidarité. Il s'adresse bien entendu également à nos dirigeants.

\section{H. Métivier} SFRP

Le nucléaire expliqué par des physiciens, Bernard Bonin coordinateur, Préface d'Étienne Klein, EDP Sciences, Coll. Introduction à, 2012, ISBN 9782-7598-0671-3, $29 €$.

Cet ouvrage s'adresse à tout citoyen concerné par les choix en matière d'énergie et d'environnement : quelle peut être la place du nucléaire à coté des autres sources d'énergie ? Le débat est largement ouvert.

H. Métivier

SFRP 